\title{
Comparison of Bragg and Polarimetric Optical Fiber Sensors for Stress Monitoring in Composite Materials
}

\author{
A.W. Domanski ${ }^{a}, \mathrm{P} \cdot \operatorname{Lesiak}^{a, *}, \mathrm{~K} \cdot \mathrm{Milenko}^{a}$, D. Budaszewski ${ }^{a}, \mathrm{M} \mathrm{Chychlowski}^{a}$,

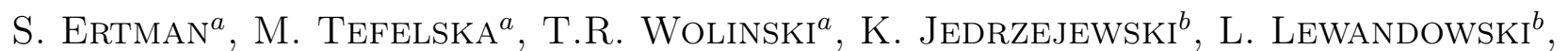 \\ W. JASIEWICZ ${ }^{b}$, J. HeLSZTYNSKI ${ }^{b}$ AND A. BOCZKOWSKA ${ }^{c}$ \\ ${ }^{a}$ Faculty of Physics, Warsaw University of Technology, Koszykowa 75, 00-662 Warsaw, Poland \\ ${ }^{b}$ Faculty of Electronics and Information Technology, Warsaw University of Technology \\ Nowowiejska 15/19, 00-665 Warsaw, Poland \\ ${ }^{c}$ Faculty of Materials Science and Engineering, Warsaw University of Technology \\ Wołoska 141, 02-507 Warsaw, Poland
}

\begin{abstract}
Composite structures are made of two or more components with significantly different physical or chemical properties and they remain separate and distinct in a macroscopic level within the finished structure. This feature allows for introducing optical fiber sensors into the composite material. These sensors can demonstrate stress distribution inside tested material influenced by external tensions. Two types of the optical fiber sensors are used as the 3D structure. One of them is based on application of fiber Bragg grating inside the core of the fiber. Longitudinal stress changes parameters of the Bragg grating and simultaneously, spectral characteristics of the light transmitted through the fiber. The second one is based on application of highly birefringent fibers which, under external stress, introduce polarization changes of the output light.
\end{abstract}

PACS numbers: 42.81.Gs, 42.81.Pa, 62.23.Pq

\section{Introduction}

The main advantages of fiber Bragg gratings (FBGs) over other optic sensor schemes are its low cost, good linearity, wavelength multiplexing capacity, resistance in harsh environments and transduction mechanism which eliminates the need for referencing as in interferometric sensors [1-5]. FBG sensor technology is now on the verge of maturity after almost three decades of active research and development in this field. Efforts are now concentrating on delivering complete FBG sensor systems including front-end electronics.

The grating will typically have a sinusoidal refractive index variation over a defined length [6]. The reflected wavelength $\left(\lambda_{\mathrm{B}}\right)$, called the Bragg wavelength, is defined by the relationship

$$
\lambda_{\mathrm{B}}=2 n \Lambda,
$$

where $n$ is the effective refractive index of the grating in the fiber core and $\Lambda$ is the grating period.

Fiber Bragg gratings can be used as sensing elements in optical fiber sensors. In a FBG sensor, the measurand causes a shift in the Bragg wavelength, $\Delta \lambda_{\mathrm{B}}$. The relative shift in the Bragg wavelength, $\Delta \lambda_{\mathrm{B}} / \lambda_{\mathrm{B}}$, due to an applied

\footnotetext{
* corresponding author; e-mail: lesiak@if.pw.edu.pl
}

strain $(\varepsilon)$ is approximately given by

$$
\frac{\Delta \lambda_{\mathrm{B}}}{\lambda}=C_{\mathrm{s}} \varepsilon,
$$

where $C_{\mathrm{s}}$ is the coefficient of strain.

The stress-induced $(\varepsilon)$ elongation of the sample in the function of the deflection is given by the following formula:

$$
\varepsilon=6 \frac{s d}{L^{2}},
$$

where $s$ - deflection, $d$ - distance between fiber layers, $L$ - length of the sample.

The change of the Bragg reflection in the function of the elongation is $1.2 \mathrm{pm}$ for $1 \mu \varepsilon$ at $1500-1600 \mathrm{~nm}$ [6].

The main disadvantage of the FBG sensors is that only a small part of the fiber is detecting stresses. In the case of the polarimetric optical fibers the whole fiber is the sensor, and because of that they could be more useful for stress monitoring particularly in composites materials.

Polarimetric optical fiber sensors based on highly birefringent (HB) polarization-maintaining fibers have focused great interest for last decades [7, 8]. In HB fibers, the difference between the phase velocities for the two orthogonally polarized modes is high enough to avoid coupling between these two modes. Fibers of this class have a built-in, well-defined, high internal birefringence obtained by designing a core and/or cladding with noncir- 
cular (mostly elliptical) geometry, or by using anisotropic stress applying parts built into the cross-section of the fiber.

The modal behavior of the lowest-order mode HB fibers under various external deformations is of special interest for sensors and device applications. A number of physical quantities can be measured on the basis of HB fibers: hydrostatic pressure, strain, vibration, temperature, acoustic wave, etc.

A symmetric deformation effect $(X)$ influences the propagation constant $\beta$ in every mode because of the changes in fiber length $L$ and the refractive indices of the core and the cladding. In a single-mode regime, this leads to changes in the phase difference $\Delta \Phi=\Delta \beta \cdot L$ between both polarizations of the fundamental $\mathrm{LP}_{01}$ mode along the fiber [1]:

$$
\frac{\delta(\Delta \Phi)}{\delta X}=\Delta \beta \frac{\partial L}{\partial X}+L \frac{\partial(\Delta \beta)}{\partial X},
$$

where $X$ stands for temperature $(T)$, pressure $(p)$ or longitudinal strain $(\varepsilon)$ defined as $\varepsilon=\Delta L / L$.

The effect of longitudinal strain on mode coupling is to modulate the relative phase retardation between the two orthogonal polarizations in the $\mathrm{LP}_{01}$ mode. The general formula describing the birefringence sensitivity to strain can be expressed in terms of an experimental parameter $T_{\varepsilon}$ describing the amount of strain $\varepsilon$ required to induce a $2 \pi$ phase shift of a polarized light observed at the output as [9]:

$$
\Delta \beta_{L}(\varepsilon)=\Delta \beta^{0}+\operatorname{sgn}\left[\frac{d(\Delta \beta)}{d \varepsilon}\right] \varepsilon \frac{2 \pi}{T_{\varepsilon} L},
$$

where $\Delta \beta_{L}^{0}$ signifies unperturbed modal (polarization) birefringence of a fiber and the function $\operatorname{sgn}[\mathrm{d}(\Delta \beta) / \mathrm{d} \varepsilon]$ has two values: +1 or -1 depending on the sign of the changes in the relative polarization birefringence with strain and $L$ is the total optical path of the fiber.

Under influence of the longitudinal strain the first term on the right-hand side of expression (4) is negligible with respect to the first, so that

$$
\delta(\Delta \Phi) \cong \frac{\partial(\Delta \beta)}{\partial \varepsilon} L \varepsilon=\frac{\partial(\Delta \beta)}{\partial \varepsilon} \delta L
$$

Hence the phase changes of the polarimetric responses are proportional to the absolute elongation $\Delta L$ and are independent of the length $L$ of the sensing region. Under the influence of a longitudinal axial strain, Eq. (6) can be approximated with the use of formula (5) in terms of the only experimental parameter $T_{\varepsilon}$ :

$$
\begin{aligned}
& \frac{\delta(\Delta \Phi)}{\delta \varepsilon}=\Delta \beta \frac{\partial L}{\partial \varepsilon}+\operatorname{sgn} \frac{\mathrm{d}(\Delta \beta)}{\mathrm{d} \varepsilon} \cdot \frac{2 \pi}{T_{\varepsilon}} \\
& \cong \operatorname{sgn} \frac{\mathrm{d}(\Delta \beta)}{\mathrm{d} \varepsilon} \cdot \frac{2 \pi}{T_{\varepsilon}} .
\end{aligned}
$$

In recent years, many experiments and researches have been carried out on laminated composites to obtain optimal mechanical properties [10-18]. Composite properties are highest in the direction of the fibers orientation. In practical application, most of the structures are not loaded in one direction, it is necessary to orient fibers in different directions. This demands evaluation of mechan- ical properties for different fiber orientations. The fiber weight fraction is an important parameter influencing the mechanical properties of composites.

\section{Materials and measurement system}

Two different 3D structures of optical fiber sensors 9 Bragg grating sensors and 15 polarimetric sensors were implemented into the composite materials. Samples were $200 \mathrm{~mm}$ long, $25 \mathrm{~mm}$ width and $2.5 \mathrm{~mm}$ thickness in both cases. Additionally, both have the same distance between fiber sensors layers: $d=1 \mathrm{~mm}$. Both types of samples were made at Faculty of Materials Science and Engineering, Warsaw University of Technology (Fig. 1).

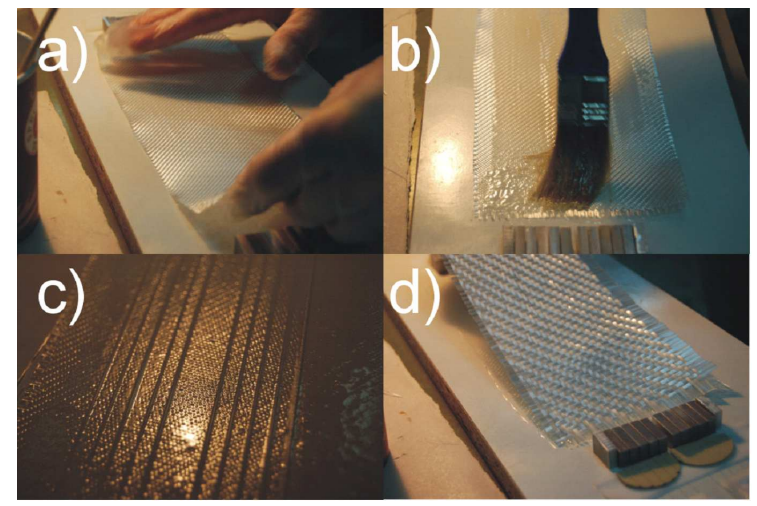

Fig. 1. Sample preparation process: (a) first 2D fabrics layer; (b) epoxy resin; (c) fiber optic sensors layer; (d) next 2D fabrics layer.

In the function of the sample bend (Fig. 2a) the biggest deformation is created at the surface. In the middle of the sample strain is almost equal to zero. The matrix of the fiber optics sensors (Fig. 2b) allows to observe the stress distribution in the deformed composite material.

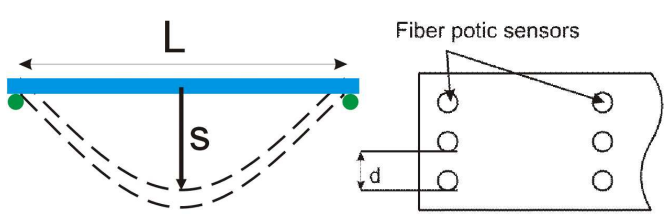

Fig. 2. Scheme of the deflection measurements (a) and fiber optic sensors distribution in the composite material (b).

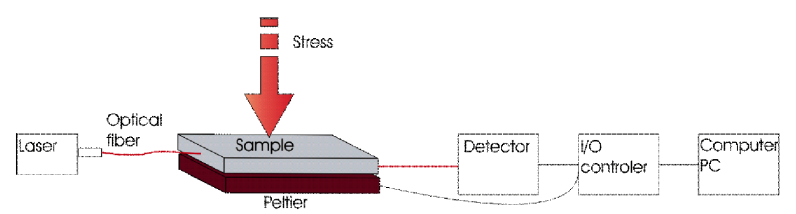

Fig. 3. Measurement setup. 
The measurement setup (Fig. 3) dedicated for fiber optic Bragg sensors consists of superluminescent INPHENIX diode as the light source: output power $3 \mathrm{~mW}$, $\lambda=1564 \mathrm{~nm}$ and FWHM $57 \mathrm{~nm}$ and the spectrum analyzer Advantest Q8384. For polarimetric sensor system measurements the polarization analyzer was used. The Peltier thermoelectric device was used for the temperature compensation.

\section{Measurement results}

The length of the fiber Bragg optic sensors was $8 \mathrm{~mm}$ each. All Bragg sensors have different reflection band. After splicing process of the 9 sensors changes during external perturbation was observed simultaneously for all fibers (Fig. 4). For polarimetric sensors influence of the bend on the output phase have to be measured separately for all 15 sensors.

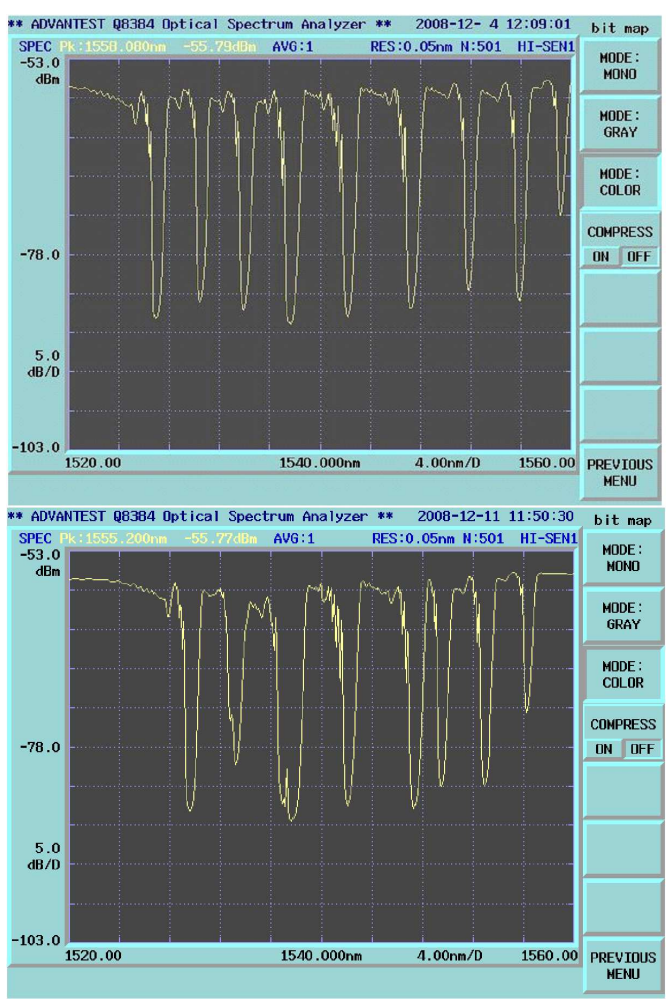

Fig. 4. Spectral characteristic of unperturbed (top) and bended (bottom) fiber Bragg sensor system.

Measurement results show that stress-induced effects in both types of the fiber optic sensors correspond to stress distribution in the deformed composite material. Both sensors exhibit the same range of elongation (strain).

However, sign of the bend-induced phase change (see Fig. 5) reveal different stress mechanisms induced in the composite material. It appeared that position of the sensing fiber (below, in the middle, or above the center of the sample) plays a predominant role in the stress-induced effects.
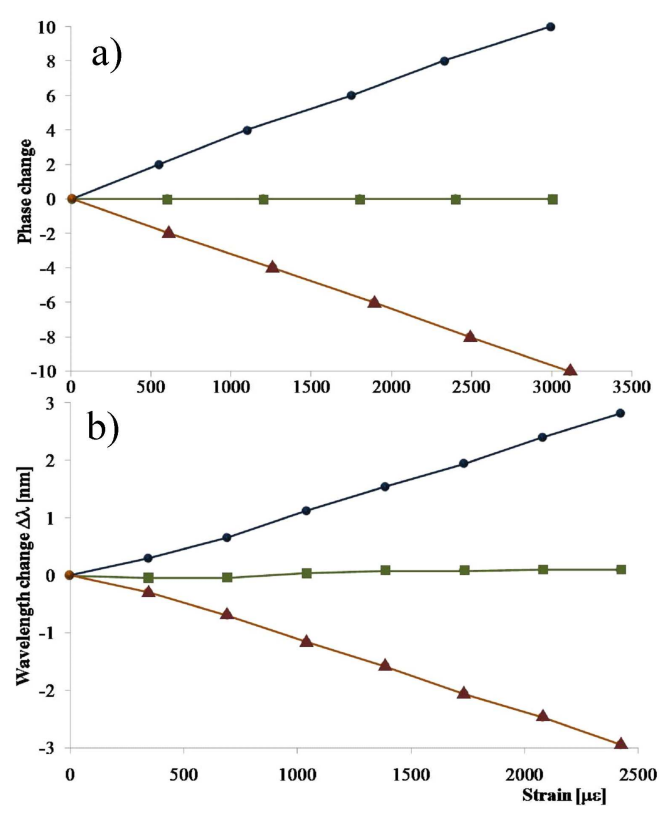

Fig. 5. Change of the polarization (a) and wavelength (b) in the function of the sample bend for fiber optic sensors placed below (triangles), in the middle (squares) and above (circles) the center of the sample.

\section{Conclusions}

Measurement results presented in this paper show that stress induced in both types of the fiber optic sensors corresponds to the stress distribution in the bended composite material. Additionally, measurements made in the same environmental conditions show that in both sensors the same range of the elongation of the fiber was detected and the specific type of the stress depends on the position of the sensing fiber - below, in the middle, or above the center of the sample.

\section{References}

[1] H.D. Simonsen, R. Paetsch, J.R. Dunphy, Proc. SPIE 1777, 73 (1992)

[2] P. Sivanesan, J. Sirkis, V. Venkat, Y.C. Shi, C.J. Reddy, S. Sankaran, Proc. SPIE 3670, 92 (1999).

[3] S. Huang, M.M. Ohn, M. LeBlanc, R.M. Measures, Smart Mater. Struct. 7, 248 (1998).

[4] Y.L. Lo, F.Y. Xiao, J. Intelligent Mater. Syst. Struct. 9, 800 (1998).

[5] V. Dewynter-Marty, P. Ferdinand, J. Intelligent Mater. Syst. Struct. 9, 785 (1998).

[6] A. Othonos, K. Kalli, Fiber Bragg Gratings Fundamentals and Applications in Telecommunications and Sensing, Artech House, London 1999.

[7] T.R. Wolinski, in: Progress in Optics, Ed. E. Wolf, North-Holland, Amsterdam 2000, p. 1.

[8] T.R. Wolinski, in: Enc. Opt. Engineering, Ed. R. Diggers, M. Dekker, New York 2003, p. 2150.

[9] W.J. Bock, A.W. Domanski, T.R. Wolinski, Appl. Opt. 29, 3484 (1990). 
[10] K.S.C. Kuang, R. Kenny, M.P. Whelan, W.J. Cantwell, P.R. Chalker, Composite Sci. Technol. 61, 1379 (2001).

[11] J.A. Guemes, J.M. Diaz-Carrilo Menendes, Proc. SPIE 3330, 264 (1998).

[12] J.A. Guemes, J.M. Diaz-Carrilo Menendes, Composite Sci. Technol. 62, 959 (2002).

[13] G. Reyes, W.J. Cantwell, Composites Sci. Technol. 60, 1085 (2000).

[14] P. Davies, W.J. Cantwell, Composites 25, 869 (1994).
[15] K. Peters, M. Studer, J. Botsis, A. Iocco, H.G. Limberger, R.P. Salathe, Proc. SPIE 3670, 195 (1999).

[16] T.K. Gangopadhyay, M. Majumder, A.K. Chakraborty, A.K. Dikshit, D.K. Bhattacharya, Sensors Actuators A 150, 78 (2009).

[17] N.C. Eaton, M.J. Curran, J.P. Dakin, H. Geiger, in: Proc. of Smart Composites, 1993, Bordeaux (France), 1993, p. 20.

[18] K. Gut, Acta Phys. Pol. A 114, A-121 (2008). 